\title{
Timelike Compton Scattering with CLAS12 at Jefferson Lab
}

Pierre Chatagnon*

IPN Orsay, for the CLAS collaboration

E-mail: chatagnoneipno.in2p3.fr

Generalized Parton Distributions (GPDs) describe the correlations between the longitudinal momentum and the transverse position of the partons inside the nucleon. At leading order, four quark-helicity conserving GPDs $(H, \tilde{H}, E$ and $\tilde{E})$ describe nucleon structure. For their direct relation to the contribution of the partons' angular momentum to the spin of the nucleon [1] and to the pressure distribution in the nucleon [2], GPDs have been at the center of many experimental programs. GPDs have been studied mainly using Deeply Virtual Compton Scattering (DVCS, $e p \rightarrow e^{\prime} p^{\prime} \gamma$ ). Here we highlight the measurement of one of the crossed processes of DVCS, Timelike Compton Scattering (TCS, $\gamma p \rightarrow e^{-} e^{+} p^{\prime}$ ), using data taken by CLAS12 at Jefferson Lab. The experimental measurement of the TCS angular asymmetry will provide new information on the real part of GPDs. This paper assesses the current status of the TCS analysis and presents preliminary results based on CLAS12 data.

Light Cone 2019 - QCD on the light cone: from hadrons to heavy ions - LC2019

16-20 September 2019

Ecole Polytechnique, Palaiseau, France

${ }^{*}$ Speaker. 


\section{Timelike Compton Scattering}

The golden process to access GPDs $[3,4,5,6]$ is DVCS $\left(e p \rightarrow e^{\prime} p^{\prime} \gamma\right)$. For large enough photon virtuality $Q^{2}$ and small $-t \ll Q^{2}$, DVCS is interpreted as an electron interacting with a single quark, which then re-emits a photon (see diagram in Figure 1). The DVCS amplitude depends on complex quantities called Compton Form Factors (CFFs) defined for the GPD $H$ as:

$$
\mathscr{H}=\sum_{q} e_{q}^{2}\left\{\mathscr{P} \int_{-1}^{1} d x H^{q}(x, \xi, t)\left[\frac{1}{\xi-x}-\frac{1}{\xi+x}\right]+i \pi\left[H^{q}(\xi, \xi, t)-H^{q}(-\xi, \xi, t)\right]\right\}
$$

and similarly for other GPDs, where $x \pm \xi$ are momentum fractions carried by the struck quark defined in Figure 1. DVCS observables measurements provide good constraints to the imaginary part of CFFs [7]. On the contrary the real part, directly linked to the pressure distribution in the nucleon [2], is much less known.

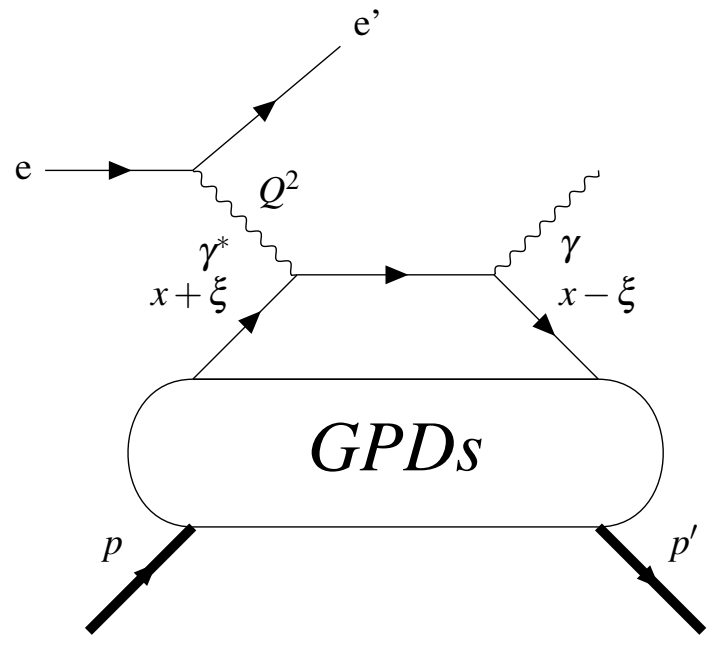

Figure 1: DVCS diagram at leading twist and leading order. At high photon virtuality $Q^{2}$ and small transferred momentum $-t=\left(p^{\prime}-p\right)^{2}$ the interaction happens at quark level. The hit quark carries a momentum fraction $x+\xi$ before the interaction, $x-\xi$ after.

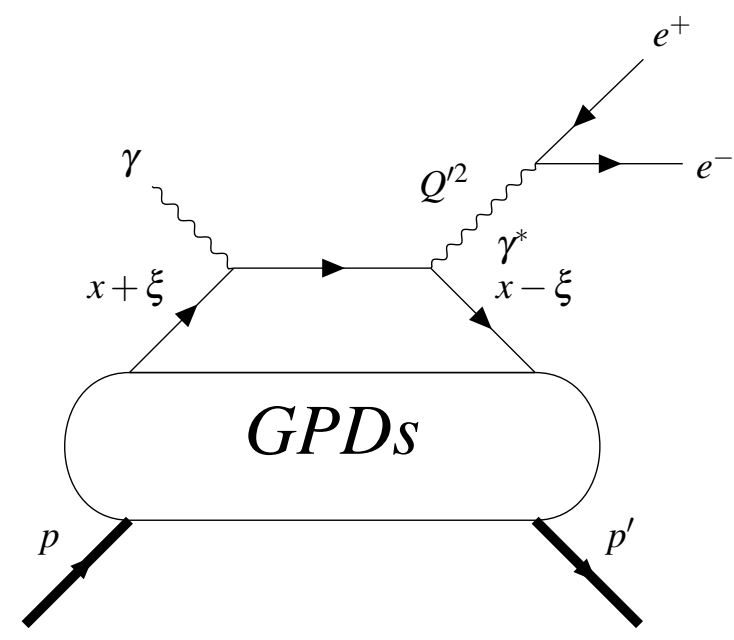

Figure 2: TCS diagram at leading twist and leading order. At high photon virtuality $Q^{\prime 2}$ and small $-t=\left(p^{\prime}-p\right)^{2}$ the interaction happens at quark level.

As for DVCS, the Timelike Compton Scattering $\left(\gamma p \rightarrow \gamma^{*} p^{\prime} \rightarrow e^{+} e^{-} p^{\prime}\right)$ amplitude depends on CFFs (see diagram in Figure 2). The cross section of the photo-production of lepton pairs $\gamma p \rightarrow$ $e^{+} e^{-} p^{\prime}$ has contributions from TCS, from the Bethe-Heitler process and from their interference. Beithe-Heitler (BH) is the decay of the incoming real photon to a lepton pair, one of the leptons then interacting with the proton. The contribution of $\mathrm{BH}$ to the total cross section is at least ten times bigger than the TCS contribution [8,9]. Thus, a direct measurement of the TCS contribution is difficult. However, the interference between BH and TCS gives access to the real part of the CFFs via the ratio $R$ proposed in [8]: 


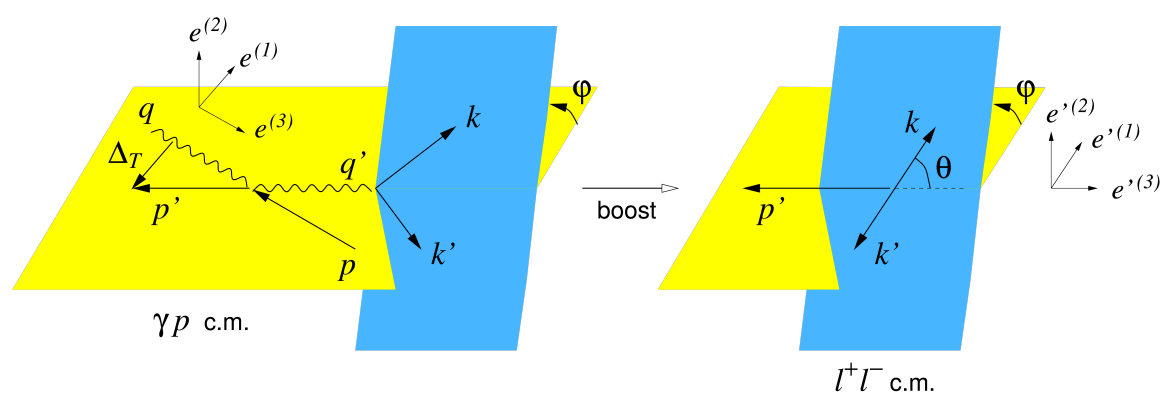

Figure 3: Definition of the kinematic variables for the $\gamma p \rightarrow e^{+} e^{-} p^{\prime}$ reaction (Figure extracted from [8]). The hadronic plane in yellow is defined by the momenta of the incoming and outgoing proton in the $\gamma p$ center-of-mass frame. The leptonic plane in blue is defined by the lepton-pair momenta.

$$
R\left(\sqrt{s}, Q^{\prime 2}, t\right)=\frac{\int_{0}^{2 \pi} d \phi \cos (\phi) \frac{d S}{d Q^{\prime 2} d t d \phi}}{\int_{0}^{2 \pi} d \phi \frac{d S}{d Q^{\prime 2} d t d \phi}}
$$

where

$$
\frac{d S}{d Q^{\prime 2} d t d \phi}=\int_{\pi / 4}^{3 \pi / 4} d \theta \frac{L}{L_{0}} \frac{d \sigma}{d Q^{\prime 2} d t d \phi d \theta}
$$

with $L=\frac{\left(Q^{\prime 2}-t\right)^{2}-b^{2}}{4}, L_{0}=\frac{Q^{\prime 4} \sin ^{2} \theta}{4}, b=2\left(k-k^{\prime}\right)\left(p-p^{\prime}\right)$, and $k, k^{\prime}, p, p^{\prime}, \theta, \phi$ are defined in Figure 3 and finally:

$$
R \propto\left[F_{1} \mathscr{H}-\xi\left(F_{1}+F_{2}\right) \tilde{\mathscr{H}}-\frac{t}{4 m_{p}^{2}} F_{2} \mathscr{E}\right],
$$

where $\mathscr{H}, \mathscr{H}$ and $\tilde{\mathscr{H}}$ are CFFs, $F_{1}$ and $F_{2}$ are Dirac and Pauli form factors and $m_{p}$ is the proton mass.

\section{Data analysis}

The angular modulation of the TCS cross section is extracted from data taken by the CLAS12 detector at Jefferson Lab. It collected data on proton target in 2018 , with a $10.6 \mathrm{GeV}$ electron beam. CLAS12 comprises tracking and Time-of-Flight (TOF) for heavy particle identification, Cherenkov detectors and electromagnetic calorimeters for lepton identification. Charge and momentum reconstruction is possible using a toroidal magnetic field. Two field polarities were used: negatively charged particles inbending and outbending with respect to the beam direction. Exploratory TCS measurements have been performed with the CLAS detector [10] and the same method is used in this analysis.

Events with a lepton pair and a proton were selected. TCS is accessible in the photo-production of a lepton pair off a proton, while in our experiment we have an electron beam impinging on a proton target. Thus, we select events where the beam electron radiated a real photon, that then interacts with a target proton. The electron is then scattered at a very low angle and stays undetected. 
The four-momentum of the scattered electron that radiated the real photon can be determined as:

$$
p_{\text {scat. } e^{-}}^{\mu}=p_{\text {beam }}^{\mu}+p_{\text {target }}^{\mu}-p_{\text {proton }}^{\mu}-p_{e^{+}}^{\mu}-p_{e^{-}}^{\mu} .
$$

The missing mass of the scattered electron $\left(M_{\text {scat. } e^{-}}^{2}=p_{\text {scat. } e^{-}}^{2}\right)$ is required to be close to zero. The transverse momentum fraction of the missing particle is cut around zero to ensure that a real photon is emitted.
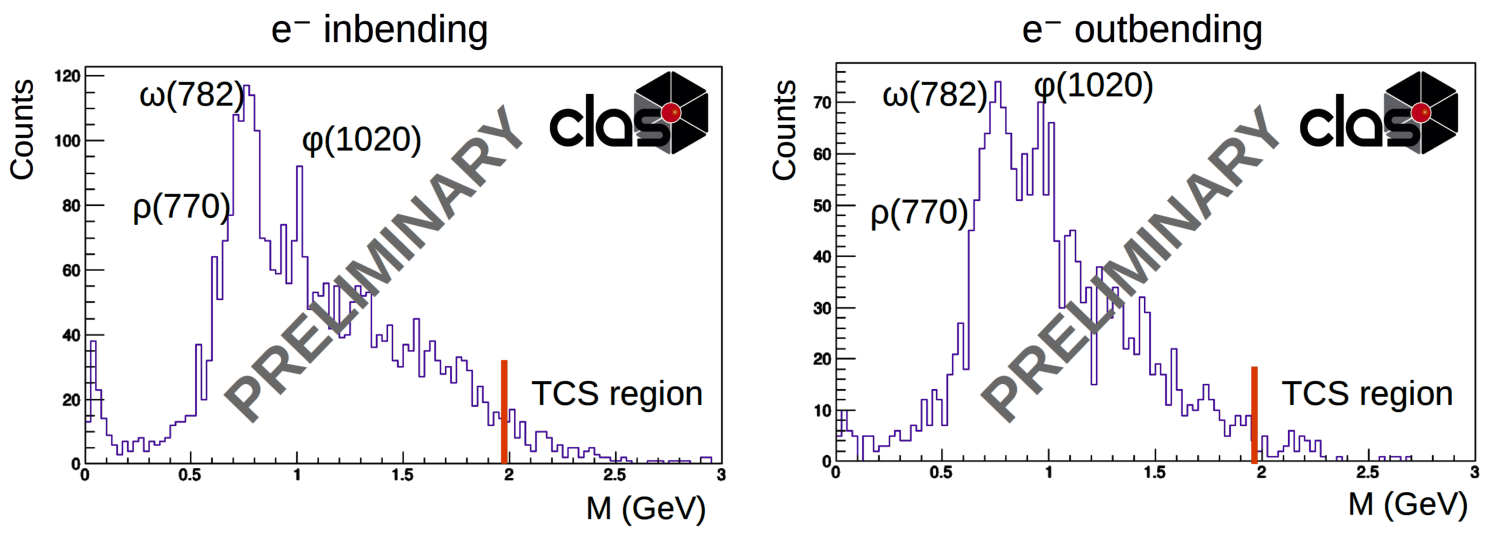

Figure 4: Lepton-pair invariant mass for the two torus magnetic field polarities. The events in the $2-3 \mathrm{GeV}$ region are selected for the measurement.

The invariant mass of the lepton pair $M_{\text {pair }}^{2}=\left(p_{e^{+}}+p_{e^{-}}\right)^{2}$ after exclusivity cuts is shown for both torus magnetic field configurations, in Figure 4. The low-mass region is dominated by vectormeson resonances $(\rho(770), \omega(782)$, and $\phi(1020))$. In the analysis, we select events in the $2-3$ $\mathrm{GeV}$ region to avoid such resonances. The data shown here corresponds to approximately $6 \%$ of the total amount of collected data currently being processed.

\section{Projected results}

From the data shown in Figure 4, 4000 events are expected in the selected mass range out of the whole data set. The achievable statistical precision with the current available data set is estimated by simulating $\gamma p \rightarrow e^{+} e^{-} p^{\prime}$ reaction weighted by $\mathrm{BH}$ and TCS/BH interference term. A GPDs double-distribution parametrization [11] is used to calculate the interference weight. The strength of the D-term is varied with a multiplying factor $\kappa$ as:

$$
H(x, \xi, t)=H_{D D}(x, \xi, t)+\kappa \frac{1}{N_{f}} \Theta(\xi-|x|) D\left(\frac{x}{\xi}, t\right)
$$

where $H_{D D}(x, \xi, t)$ is the parametrization of the GPD, $N_{f}$ is the number of active flavors and $\kappa$ is the D-term strength (see [8]). The R ratio is obtained in the CLAS12 acceptance as a function of $-t$ for three different values of $\kappa$ and for BH-only weighted events. Results are displayed in Figure 5, including projected error bars. Our measurement is expected to provide an insight on the strength of the D-term. 


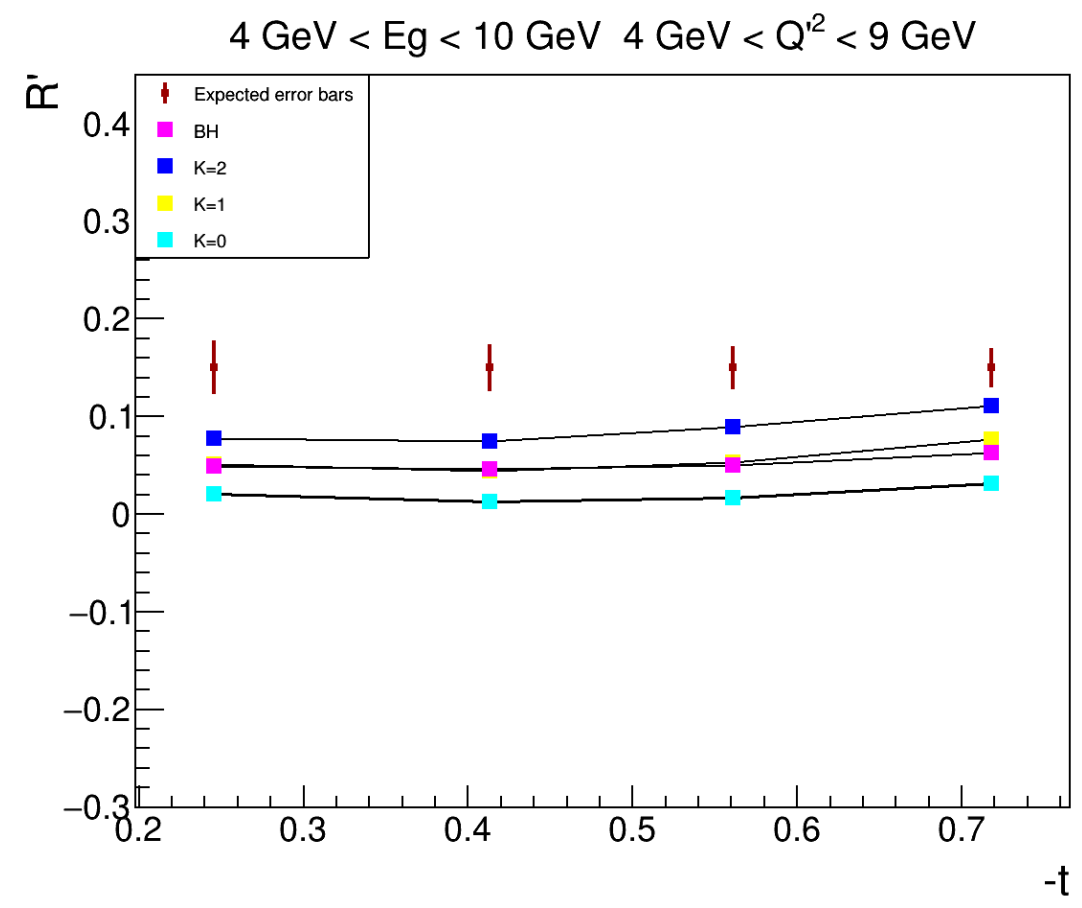

Figure 5: The ratio $R$ calculated as a function of transferred momentum $t$ in the CLAS12 acceptance for different strengths of the D-term and for BH-only weighted events. The error bars correspond to the expected statistical errors achievable for the current data set taken by CLAS12 (values are arbitrary).

\section{References}

[1] Xiangdong Ji. Gauge-invariant decomposition of nucleon spin. Phys. Rev. Lett., 78:610-613, Jan 1997.

[2] V. Burkert, L. Elouadrhiri, and F. Girod. The pressure distribution inside the proton. Nature, 557, 05 2018.

[3] A. V. Radyushkin. Nonforward parton distributions. Phys. Rev., 56:5524-5557, Nov 1997.

[4] Michel Guidal, Hervé Moutarde, and Marc Vanderhaeghen. Generalized parton distributions in the valence region from deeply virtual compton scattering. Reports on Progress in Physics, 76(6):066202, 2013.

[5] M. Diehl. Generalized parton distributions. Physics Reports, 388(2):41 - 277, 2003.

[6] Markus Diehl. Introduction to gpds and tmds. The European Physical Journal, 52(6):149, Jun 2016.

[7] Nicole d'Hose, Silvia Niccolai, and Armine Rostomyan. Experimental overview of deeply virtual compton scattering. The European Physical Journal, 52(6):151, Jun 2016.

[8] E.R. Berger, M. Diehl, and B. Pire. Timelike compton scattering: exclusive photoproduction of lepton pairs. The European Physical Journal, 2, 2002.

[9] Boer, M., Guidal, M., and Vanderhaeghen, M. Timelike compton scattering off the proton and generalized parton distributions. Eur. Phys. J., 51(8):103, 2015. 
[10] R. Paremuzyan. Timelike Compton Scattering. PhD thesis, Yerevan Physics Institute, 2010.

[11] A. V. Radyushkin. Double distributions and evolution equations. Phys. Rev., 59:014030, Dec 1998. 